Article

\title{
Study of the Influence of Different Yeast Strains on Red Wine Fermentation with NIR Spectroscopy and Principal Component Analysis
}

\author{
Antonio Domenico Marsico, Rocco Perniola, Maria Francesca Cardone $₫$, Matteo Velenosi, \\ Donato Antonacci, Vittorio Alba and Teodora Basile *(D) \\ Council for Agricultural Research and Economics-Research Centre for Viticulture and Enology, Turi (BA) 70010, \\ Italy; adomenico.marsico@crea.gov.it (A.D.M.); rocco.perniola@crea.gov.it (R.P.); \\ mariafrancesca.cardone@crea.gov.it (M.F.C.); matteo.velenosi@tiscali.it (M.V.); \\ donato.antonacci@crea.gov.it (D.A.); vittorio.alba@crea.gov.it (V.A.) \\ * Correspondence: teodora.basile@crea.gov.it; Tel.: +39-080-891-5711
}

Received: 3 October 2018; Accepted: 27 October 2018; Published: 31 October 2018

check for updates

\begin{abstract}
Alcoholic fermentation is a key step in wine production. Indeed, a wide range of compounds, which strongly affect the sensory properties of wine, is produced during this process. While Saccharomyces cerevisiae yeast cultures are commonly employed in winemaking to carry on the fermentation process, some non-Saccharomyces species have recently gained attention due to their ability to produce various metabolites of oenological interest. The use of different yeasts strains usually results in wines with different sensory properties, despite being obtained from the same grape variety. In this paper, we tested the feasibility of using near-infrared spectroscopy (NIR) to discriminate among red wines from three different grape varieties produced with pure $S$. cerevisiae or by mixed fermentation with a promising non-Saccharomyces yeast, namely the Starmeriella bacillaris, which usually yields wines with significant amounts of glycerol and low levels of ethanol, acetic acid, and acetaldehyde. A principal component analysis (PCA) performed on the NIR spectra was used to search for differences in the samples. The NIR results have been compared with both basic wine parameters and sensory analysis data.
\end{abstract}

Keywords: alcoholic fermentation; Saccharomyces cerevisiae; Starmerella bacillaris; NIR spectroscopy; Negramaro; Primitivo; Aleatico nero

\section{Introduction}

In recent years, a steady growth of both wine consumption and trade has been observed worldwide [1]. With wine being a valuable commodity, the wine market is a highly competitive arena, demanding a challenging search for novel features and improved wine qualities. The composition of a wine resulting from a spontaneous fermentation of grape juice carried out by indigenous yeasts is difficult to predict since the interaction of these yeasts belonging to different genera and species during fermentation depends on several factors. This results in unpredictable changes in the wine quality from one year to another or from one region to another [2]. Due to the influence of yeast strains on the composition and sensory quality of a wine, the selection of yeast strains for the alcoholic fermentation step is of paramount importance in any winemaking. Nowadays, selected pure Saccharomyces cerevisiae starter cultures are commonly employed to carry out the fermentation process, since they are able to suppress the wild microflora and dominate the fermentation process [3]. However, a growing number of scientific publications have reported the use of selected strains of non-Saccharomyces yeasts (pure or in combination), and have analyzed their influence on the composition and quality of wine on different varieties of grapes, trying to exploit the relationship between the presence of these strains and the type of wine produced [3,4]. Among the 
non-Saccharomyces yeasts, Starmerella bacillaris has recently received attention since it not only is able to grow at high concentrations of sugars and low temperatures [5], but also it produces significant amounts of glycerol and low levels of ethanol, acetic acid, and acetaldehyde [6,7]. Moreover, it has been reported that this yeast is able to impart novel positive features to the sensory profile of the wine. Indeed, mixed fermentation has effectively modified the aromatic profiles of wines produced from non-floral grape varieties like Barbera [8]. Twelve winemakings of well-known red wine grape varieties (i.e., Negramaro, Primitivo and Aleatico nero) have been performed, employing pure starter cultures of $S$. cerevisiae and mixed fermentations with $S$. bacillaris.

In order to discriminate among wines produced with pure or mixed starter cultures, a number of chemical analyses are usually needed. Those analyses are time-consuming, require sophisticated analytical techniques (e.g., HPLC, GC-MS), and are expensive. Near-infrared (NIR) spectroscopy is a technique already employed in food and beverage analysis to determine several parameters in a single, rapid, and non-destructive steps. This analytical technique has been successfully applied to wine analysis for the qualitative and quantitative characterization of its main compounds [9-11]. In this paper, we tested the ability of this technique to discriminate among different wines produced with pure and mixed fermentation. A large number of organic molecules meet the requirements for NIR spectroscopic analysis. The vast amount of NIR spectral information often results in very complex spectra with a large number of overlapping absorption signals. In order to extract information from those spectra, multivariate techniques are required. Instead of searching for specific compounds responsible for differences in wine composition in the spectra, we performed a principal component analysis (PCA) using the NIR spectra as variables. PCA is one of the most popular classification methods utilized in life sciences. This method is employed to reduce the number of variables (scores) by capturing variance in the samples. Indeed, significant information from the NIR spectra is retained in a set of new, orthogonal variables called principal components (PCs), which then are used as novel axes in the PCA plots [12]. In a PCA plot, it is possible to visualize the most important information from a given multivariate data set. This analysis allowed us to find sample groupings for samples from different winemakings, without any a priori knowledge of the differences. The PCA results have been compared to chemical analysis data of basic wine parameters in order to understand if the main constituents of wine (e.g., ethanol) were responsible for the differences found in the NIR spectra. Metabolites responsible for the differentiation were putatively identified using NIR spectra signals, or regions with high loading onto the PCA axes, attributable to known compounds. The statistical analysis performed on sensory data confirmed a lack of significant differences for wines which were not differentiated by NIR analysis.

In this paper, the potential of NIR spectroscopy as a rapid screening technique to discriminate wines produced with different yeast strains was investigated. The differences in the metabolic profiles of wines, which were responsible for NIR differentiation, were putatively identified.

\section{Materials and Methods}

\subsection{Grape Characteristics and Winemaking}

A total of twelve micro-fermentations of Negramaro, Primitivo and Aleatico nero varieties have been performed during the production of the 2018 vintage in the Apulia region (Southern Italy). About $20 \mathrm{~kg}$ of bunches per each winemaking were harvested at technical maturity. Two strains of S. cerevisiae (E16 and E138) and the non-Saccharomyces yeast S. bacillaris FA18 were used. All the yeasts were kindly provided by the Institut Universitaire de la Vigne et du Vin, University of Burgundy, Dijon, France. For each variety, two types of winemaking have been performed: the first by inoculating a 50:50 ratio of two strains of $S$. cerevisiae, the second through a mixed fermentation, i.e., initial inoculation of the must with $S$. bacillaris strain, followed after 2 days by inoculation with a 50:50 ratio of the two strains of $S$. cerevisiae. During the fermentation process, three fillings were performed manually every $7-8 \mathrm{~h}$ during the first $3 / 4$ of the consumption of sugars, and then two fillings every $10-12 \mathrm{~h}$ until 
total consumption of the sugars. The temperature was kept constant at $25^{\circ} \mathrm{C}$. Finally, musts were manually pressed in a wine press and stored at $10^{\circ} \mathrm{C}$ until bottling (two-three days). Each of the three winemaking procedures was performed in duplicate (samples labeled with A and B), thus for each winemaking procedure, two bottles have been analyzed. Data obtained with primary methods for wine's basic parameters, alcohol content, pH, volatile, and titratable acidity, are shown in Table 1.

Table 1. Wine basic parameters.

\begin{tabular}{|c|c|c|c|c|}
\hline Samples & Alcohol (\%Vol) & Volatile Acidity $^{{ }^{+}}$(g/L) & $\mathrm{pH}$ & Titratable Acidity $\ddagger(g / L)$ \\
\hline \multicolumn{5}{|c|}{ Primitivo } \\
\hline E16A & 15.4 & $0.54 \pm 0.01$ & $3.10 \pm 0.01$ & $7.71 \pm 0.03$ \\
\hline E16B & 15.6 & $0.42 \pm 0.01$ & $3.14 \pm 0.02$ & $7.90 \pm 0.02$ \\
\hline E138A & 14.4 & $0.41 \pm 0.00$ & $3.23 \pm 0.02$ & $7.5 \pm 0.01$ \\
\hline E138B & 15.3 & $0.44 \pm 0.00$ & $3.17 \pm 0.02$ & $7.75 \pm 0.02$ \\
\hline FA18A & 15.1 & $0.45 \pm 0.00$ & $3.20 \pm 0.02$ & $7.60 \pm 0.02$ \\
\hline FA18B & 15.3 & $0.47 \pm 0.00$ & $3.29 \pm 0.01$ & $7.73 \pm 0.05$ \\
\hline \multicolumn{5}{|c|}{ Negroamaro } \\
\hline E16A & 12.5 & $0.36 \pm 0.00$ & $3.36 \pm 0.01$ & $6.10 \pm 0.02$ \\
\hline E16B & 12.1 & $0.35 \pm 0.00$ & $3.43 \pm 0.01$ & $6.02 \pm 0.03$ \\
\hline E138A & 12.4 & $0.33 \pm 0.00$ & $3.31 \pm 0.01$ & $6.06 \pm 0.03$ \\
\hline E138B & 12.6 & $0.42 \pm 0.01$ & $3.27 \pm 0.01$ & $6.12 \pm 0.05$ \\
\hline FA18A & 12.2 & $0.44 \pm 0.01$ & $3.37 \pm 0.01$ & $6.62 \pm 0.03$ \\
\hline FA18B & 12.3 & $0.32 \pm 0.00$ & $3.32 \pm 0.01$ & $6.19 \pm 0.00$ \\
\hline \multicolumn{5}{|c|}{ Aleatico nero } \\
\hline E16A & 12.1 & $0.35 \pm 0.00$ & $3.22 \pm 0.01$ & $5.59 \pm 0.06$ \\
\hline E16B & 11.8 & $0.38 \pm 0.01$ & $3.28 \pm 0.01$ & $5.61 \pm 0.03$ \\
\hline E138A & 11.9 & $0.36 \pm 0.00$ & $3.31 \pm 0.01$ & $5.67 \pm 0.05$ \\
\hline E138B & 11.9 & $0.35 \pm 0.00$ & $3.20 \pm 0.00$ & $5.50 \pm 0.02$ \\
\hline FA18A & 11.6 & $0.39 \pm 0.01$ & $3.25 \pm 0.01$ & $6.15 \pm 0.06$ \\
\hline FA18B & 11.7 & $0.47 \pm 0.02$ & $3.18 \pm 0.02$ & $6.21 \pm 0.03$ \\
\hline
\end{tabular}

${ }^{\dagger}$ expressed as acetic acid; ${ }^{\ddagger}$ expressed as tartaric acid.

\subsection{FT-NIR Instrument and Chemometric Analysis}

Fourier Transform Near Infrared (FT-NIR) analyses were performed with a Bruker TANGO FT-NIR spectrometer, and spectra acquisition range was 12,000-3600 $\mathrm{cm}^{-1}\left(8 \mathrm{~cm}^{-1}\right.$ resolution and 64 scans for both background and sample) at a controlled temperature $\left(25 \pm 1^{\circ} \mathrm{C}\right)$ and constant humidity. The OPUS/QUANT software (Bruker Optik GmbH, Ettlingen, Germany) version 2.0 was used for spectral acquisition and chemometric analysis. A blank was measured prior to the acquisition of each sample (measured three times). Any water present in the samples dominates spectra of natural products, and for this reason, quantitative analysis often relies on minor changes in spectra [13]. PCA was applied to spectral data, as an exploratory tool, to differentiate wines based on their metabolic compositions resulting from the different fermentation processes performed. PCA identifies orthogonal directions of maximum variance in the original dataset in decreasing order and projects the data onto a lower-dimensionality space formed by a subset of the highest-variance components. The orthogonal directions are linear combinations of the original variables, and each component explains part of the total variance of the data [14]. Before the PCA analysis, a mean centering of all the spectra was performed, and outliers were identified and removed.

Moreover, a PCA was carried out with R Statistical Software (R Core Team (2013), R Foundation for Statistical Computing, Vienna, Austria), employing the main wine parameters as variables. 


\subsection{Sensory Analysis}

The panel test ( 7 healthy subjects, 2 women and 5 men) was composed of already experienced tasters chosen among the staff of the CREA-VE of Turi. The tasting session was performed following the ISO indications [15-17]. Prior to the evaluation, five experienced wine judges selected twelve sensorial descriptors to characterize the wines. Twelve attributes were selected to describe the wines: visual characteristics (color intensity), odor descriptors (fresh fruits, mature fruits, floral, herbal and spicy character), and taste and tactile/textural descriptors (acidity, astringency, body, alcohol, and persistence), together with an overall rating of each wine. The scoring sheets were anchored with the respective low perception for score 1 and high perception for score 10, and panelists could choose only integer values $[18,19]$. The significance of the sensory analysis was assessed performing the Kruskal-Wallis non-parametric test, followed by the Dunn test as a post hoc test, using a $p$-value $<0.05$, considering each judge as a replicate. All statistical analyses were performed with $R$ Statistical Software (R Core Team (2013), R Foundation for Statistical Computing, Vienna, Austria).

\section{Results}

\subsection{Basic Parameters and NIR Analysis}

Six samples of wine produced with pure or mixed fermentation from each of the three varieties were analyzed by NIR. In the unprocessed NIR spectra the main wine compounds, i.e., water and ethanol, dominate the spectrum and often overshadow minor compounds with similar functional groups, which show peaks in the same regions. Absorption bands with high absorption units (e.g., some water signals) are automatically cut out by the instrument. They should not be used since they are characterized by very small light intensities, and the resulting signals contain more noise [20]. NIR spectra of fruits, vegetables, and their juices, with a high percentage of water, show absorption bands related to water around $6800 \mathrm{~cm}^{-1}$ and at $5000 \mathrm{~cm}^{-1}$ [21]. Water is the main molecule responsible for those signals, but it is not the only one. Other molecules with $\mathrm{O}-\mathrm{H}$ groups contribute to the same (or very close) area of the spectrum, e.g., around $5000 \mathrm{~cm}^{-1}$ of glucose and ethanol also show peaks [22].

The grouping in the PCA plot obtained from the spectra of all the three varieties (Figure 1A) shows that Primitivo samples are grouped on the left side, while it is not possible to separate samples from the other two varieties. It is clear that samples are grouped by the variety and not by the different yeasts employed in the winemaking. The loadings (Figure 1B) of this PCA analysis show how the wavelengths mainly responsible for the variance in the data set are around $7000 \mathrm{~cm}^{-1}$, $5300 \mathrm{~cm}^{-1}$, and $488-4200 \mathrm{~cm}^{-1}$, which correspond respectively to the first overtones of $\mathrm{COH}$ groups, the first overtones of $\mathrm{CH}_{2}$ and $\mathrm{CH}_{3}$ groups, and $\mathrm{COH}$ combination (stretching and bending) vibrations. In the NIR spectra, there was a strong overlap of signals of functional groups belonging to different molecules. Thus, a specific attribution is not an easy task. Nevertheless, the strong signal around $700 \mathrm{~cm}^{-1}$ can be mainly attributed to $\mathrm{OH}$ vibrations of two of the main compounds found in wine, which are water and ethanol. In the spectra of pure ethanol, it was observed that the absorbance changes with the ethanol concentration [9]. Indeed, the three wines showed very different ethanol contents (similar for Negroamaro and Aleatico and higher for Primitivo) which might explain the grouping in the PCA plot. Clearly, due to this strong difference in one of the main components of the samples, ascertaining the differences in terms of other (minor) compounds was not possible. These hypotheses were confirmed by the groupings found in the PCA performed using chemical analysis data as variables (Figure 2), in which Primitivo samples were grouped together due to their acidic composition and higher content of ethanol. Indeed, the signals linked to the main acids found in wine (tartaric, malic, acetic and lactic acid) in a NIR spectrum are too close to those of ethanol, which overshadows them with its higher content. 


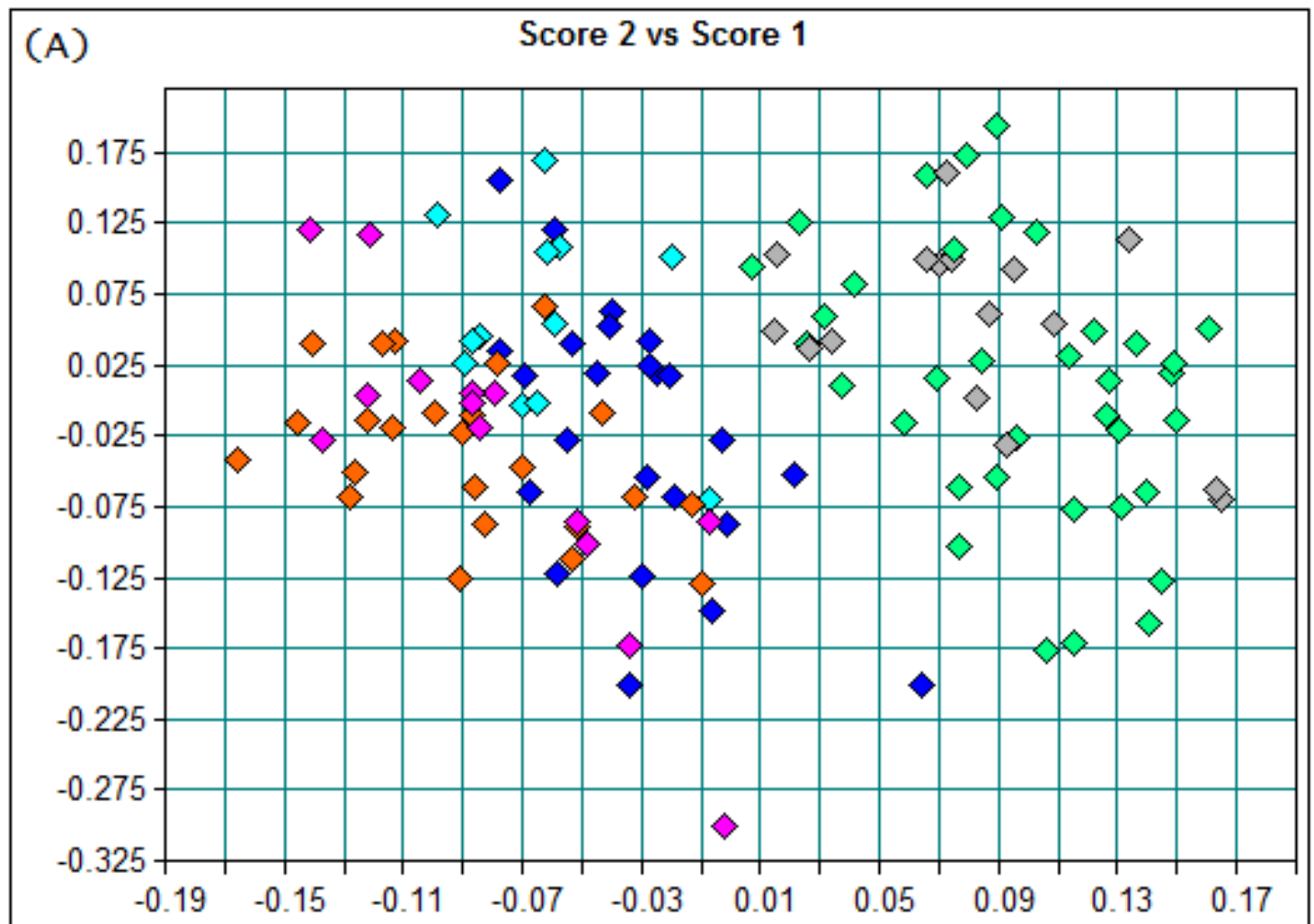

(B)

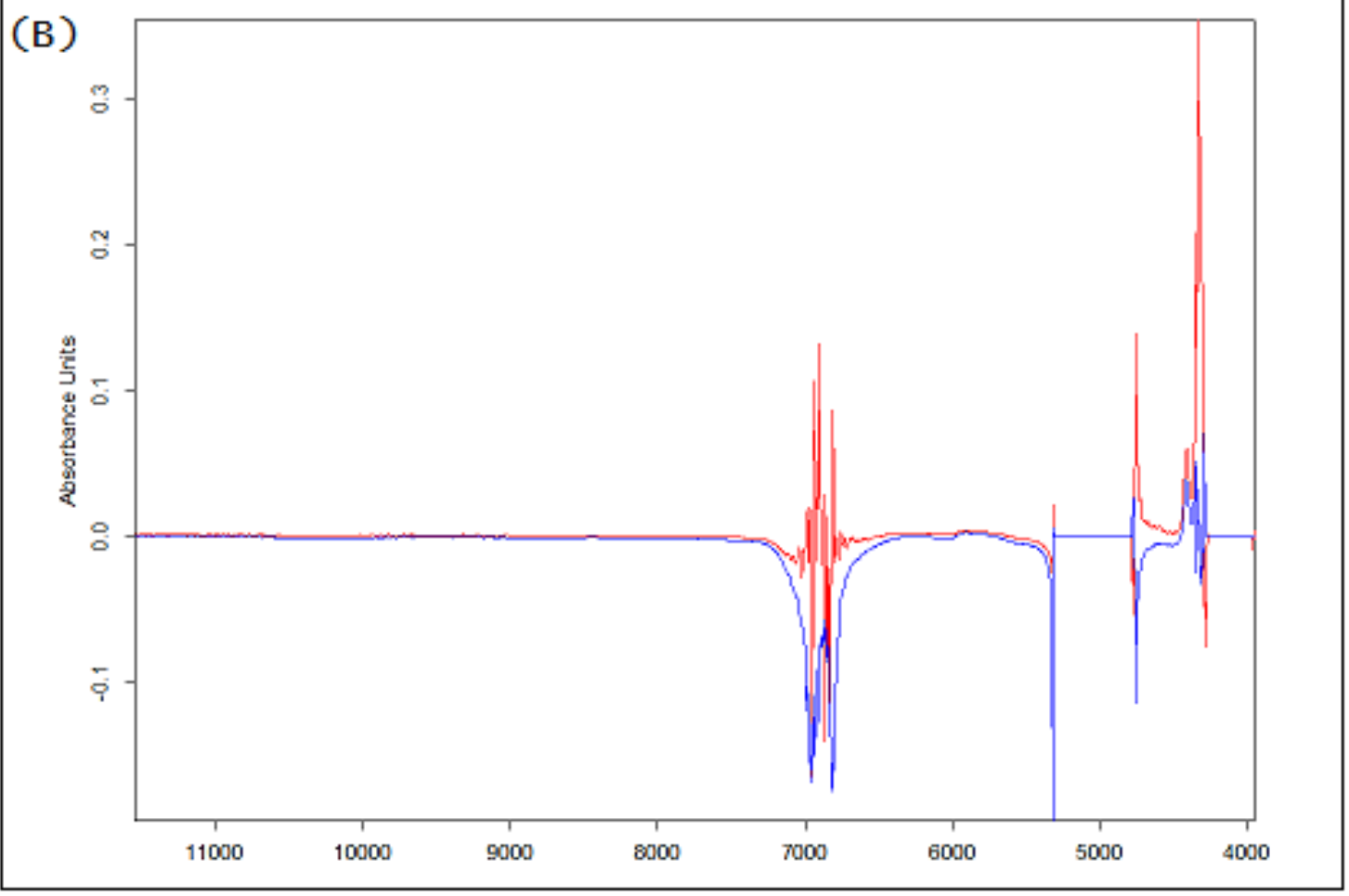

Figure 1. (A) Principal component analysis (PCA) plots of all the samples: Negroamaro pure (in blue) and mixed (in cyan), Primitivo pure (in green) and mixed (in gray), Aleatico nero pure (in magenta) and mixed (in orange). (B) Loadings: Score one (y-axis) is in blue while score two (x-axis) is in red. 


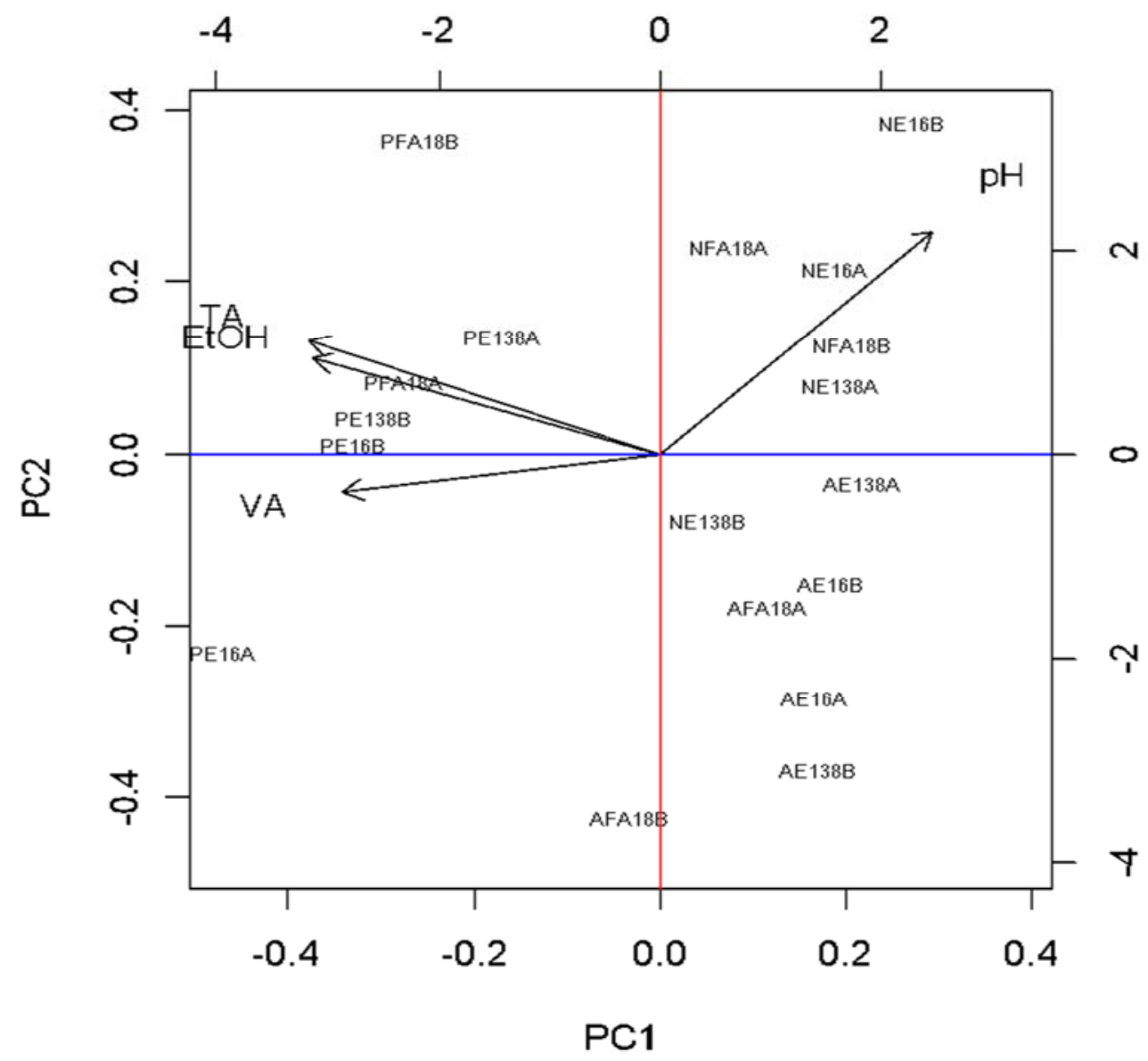

Figure 2. PCA plot of all the samples (N-Negroamaro, A-Aleatico and P-Primitivo) with basic parameters used as variables: $\mathrm{pH}$, volatile acidity (VA), titratable acidity (TA), and alcohol (EtOH).

In order to minimize the influence of ethanol and water, we decided to investigate each variety on its own. The NIR analysis of the Negroamaro samples, with close attention to ethanol content, was able to discriminate between the wines obtained with S. cerevisiae and those obtained with non-Saccharomyces (Figure 3A). The difference between the NIR and chemical PCAs of Negroamaro suggest that the variables employed to build the chemical data PCA (Figure 4) were not responsible for the grouping found in the NIR PCA. Negroamaro samples have similar ethanol content, thus, even if the loadings of NIR PCA are in similar regions of the spectra (Figure 3B), compared to those of NIR PCA of all the samples together, the loadings in the regions $6500-7000 \mathrm{~cm}^{-1}$ and $4500-4300 \mathrm{~cm}^{-1} \mathrm{can}$ be attributable to compounds other than ethanol. We attempted an interpretation based on the known NIR absorbance signals of metabolites commonly present in wine, focusing only on the NIR spectral regions responsible for wine differentiation (the regions with high loadings on PCA axes). It has been previously reported that wines from non-Saccharomyces produce different metabolites (e.g., are found to be more fruity) or produce them in different concentration (e.g., glycerol) [23]. Indeed, in the NIR PCA loadings, important spectral regions are those related to glycerol $\left(4400,5300,7200 \mathrm{~cm}^{-1}\right)$ [22]. This led us to hypothesize that glycerol content might be one of the factors responsible for the differentiation found between pure and mixed fermentation samples. 


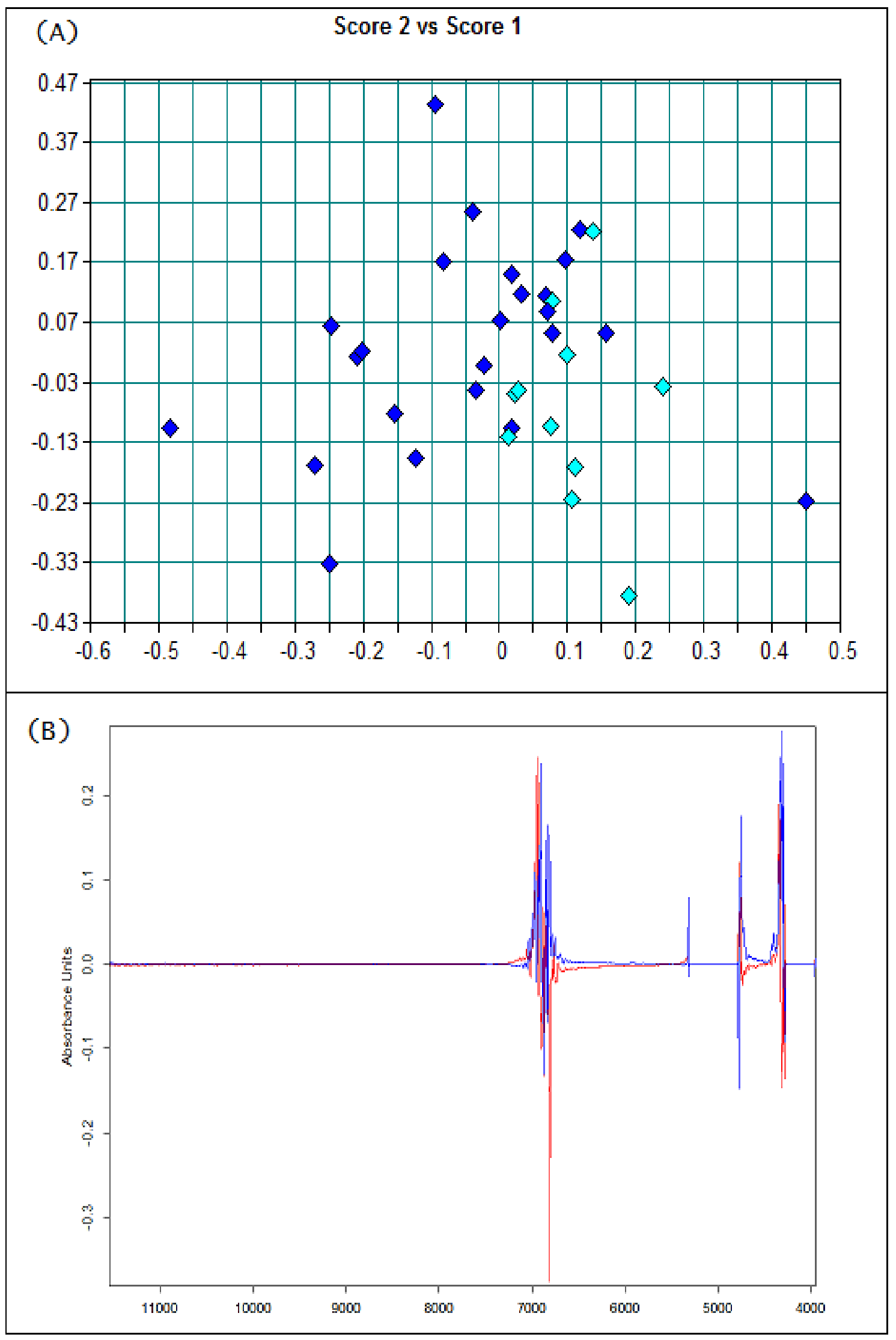

Figure 3. Mono varietal PCA plot of Negroamaro samples from near-infrared spectroscopy (NIR) data; (A) pure (in blue) and mixed (in cyan) fermentation; (B) PCA loadings. 


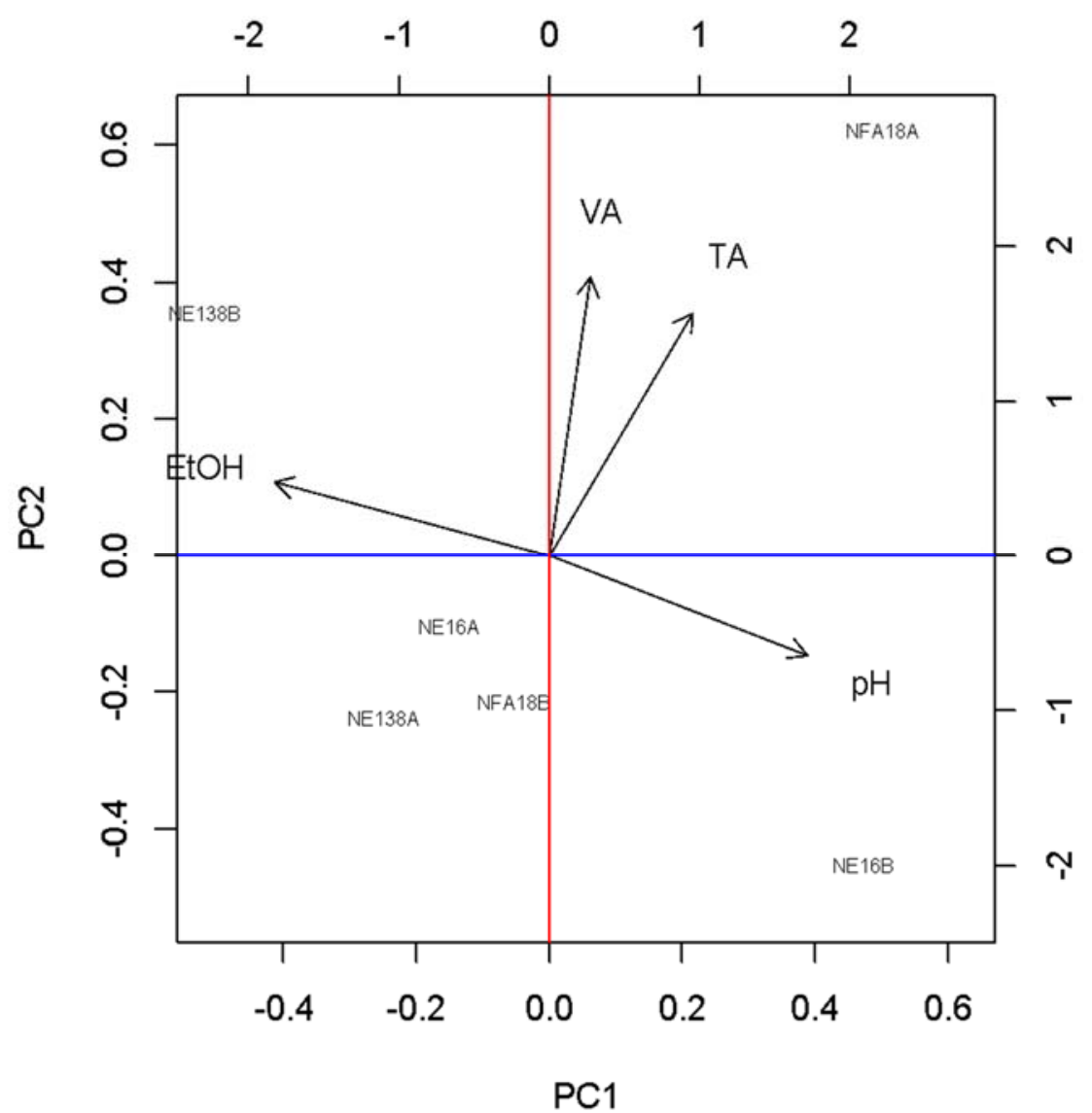

Figure 4. Mono varietal PCA plot of Negroamaro (N) wines from chemical analyses.

For Aleatico nero wines, there is not a clear separation between pure and mixed fermentation samples in the PCA plot obtained with NIR data (Figure 5), while in the PCA plot built with chemical data, the pure and mixed fermentation samples are clearly separated (Figure 6). Here, ethanol content is again not the main variable responsible for sample differentiation. Despite the specific nature of compounds responsible for differences among wines, it was not possible to discriminate between pure and mixed fermentation wines with NIR spectroscopy. 


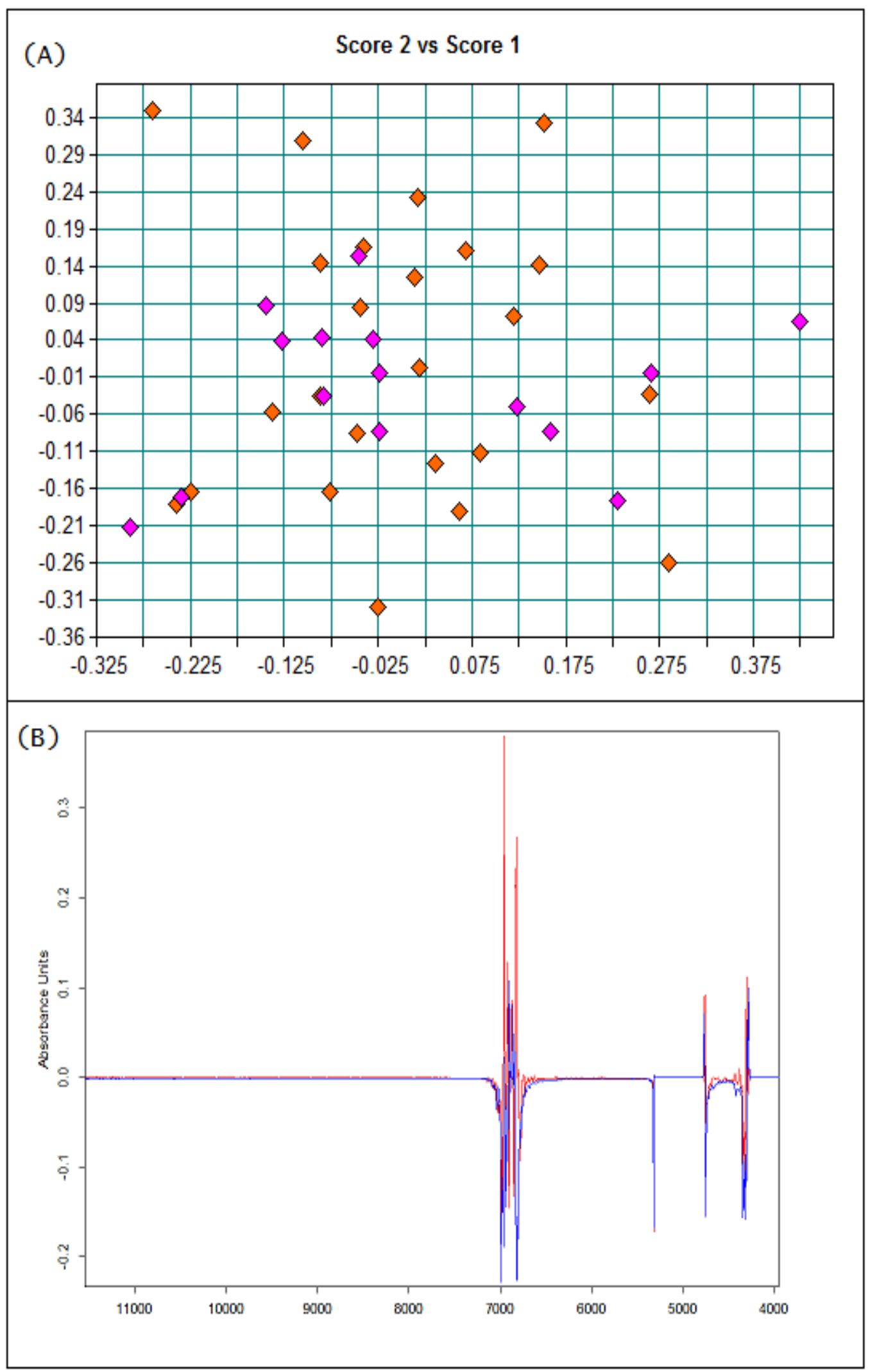

Figure 5. Mono varietal PCA plot of Aleatico nero samples from NIR data (A) pure (in magenta) and mixed (in orange); (B) PCA loadings. 


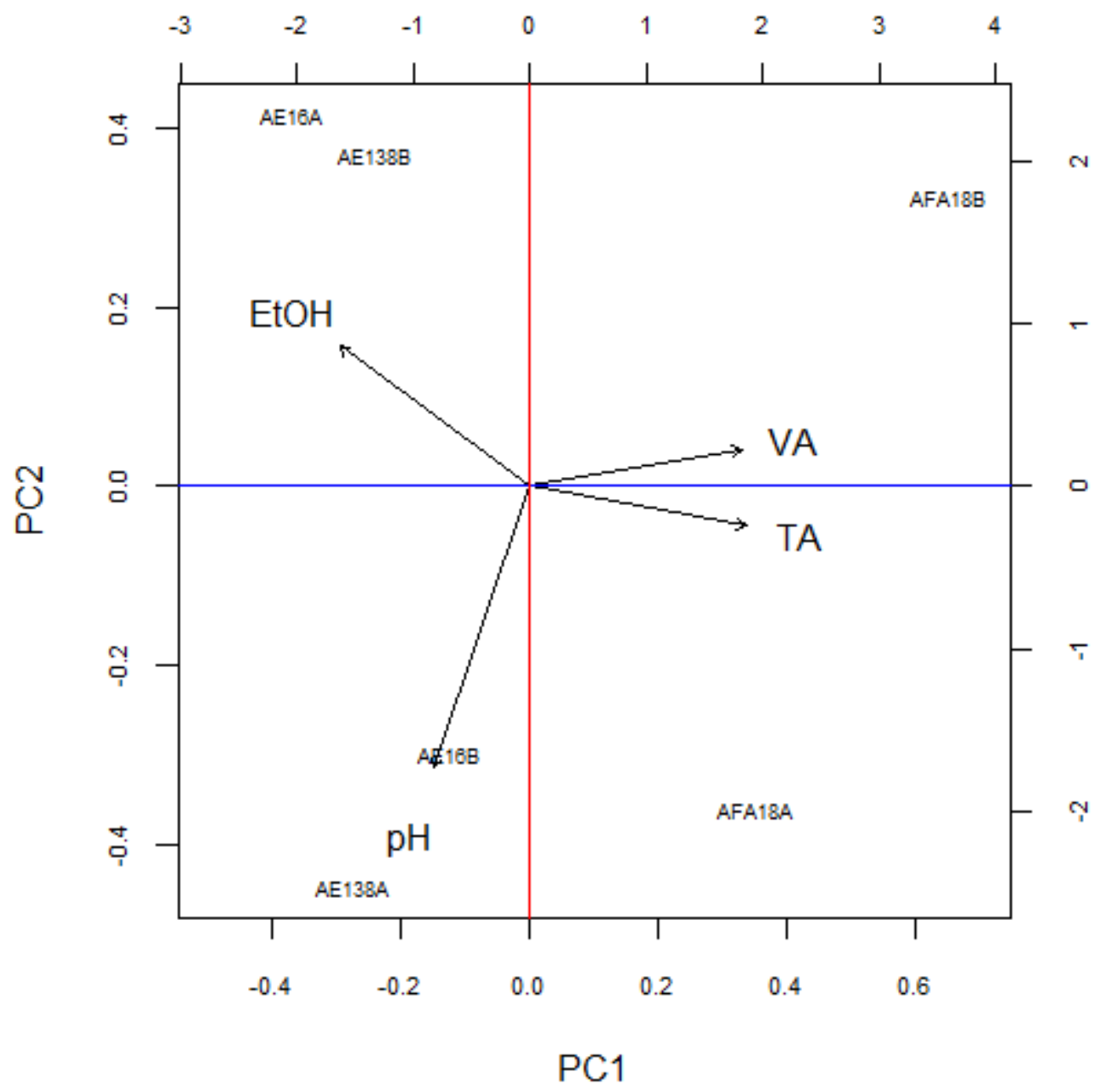

Figure 6. Mono varietal PCA plot of Aleatico nero (A) wines from chemical analyses.

In both the PCAs of the Primitivo samples, there is not a clear separation between pure and mixed fermentation (Figures 7 and 8). Indeed, even considering only the chemical data, there is not a net difference among all the monovarietal Primitivo wines. The numerosity of samples is a key point in NIR analysis. As for Aleatico nero wine, a larger number of samples could have improved the separation among wines and helped to identify differences. In order to understand if these results were due to a lack of discriminant capacity of the method employed or rather due to a lack of significant differences in wines composition, we investigated the perceived difference among pure and mixed fermentation wines by performing a sensory analysis. 


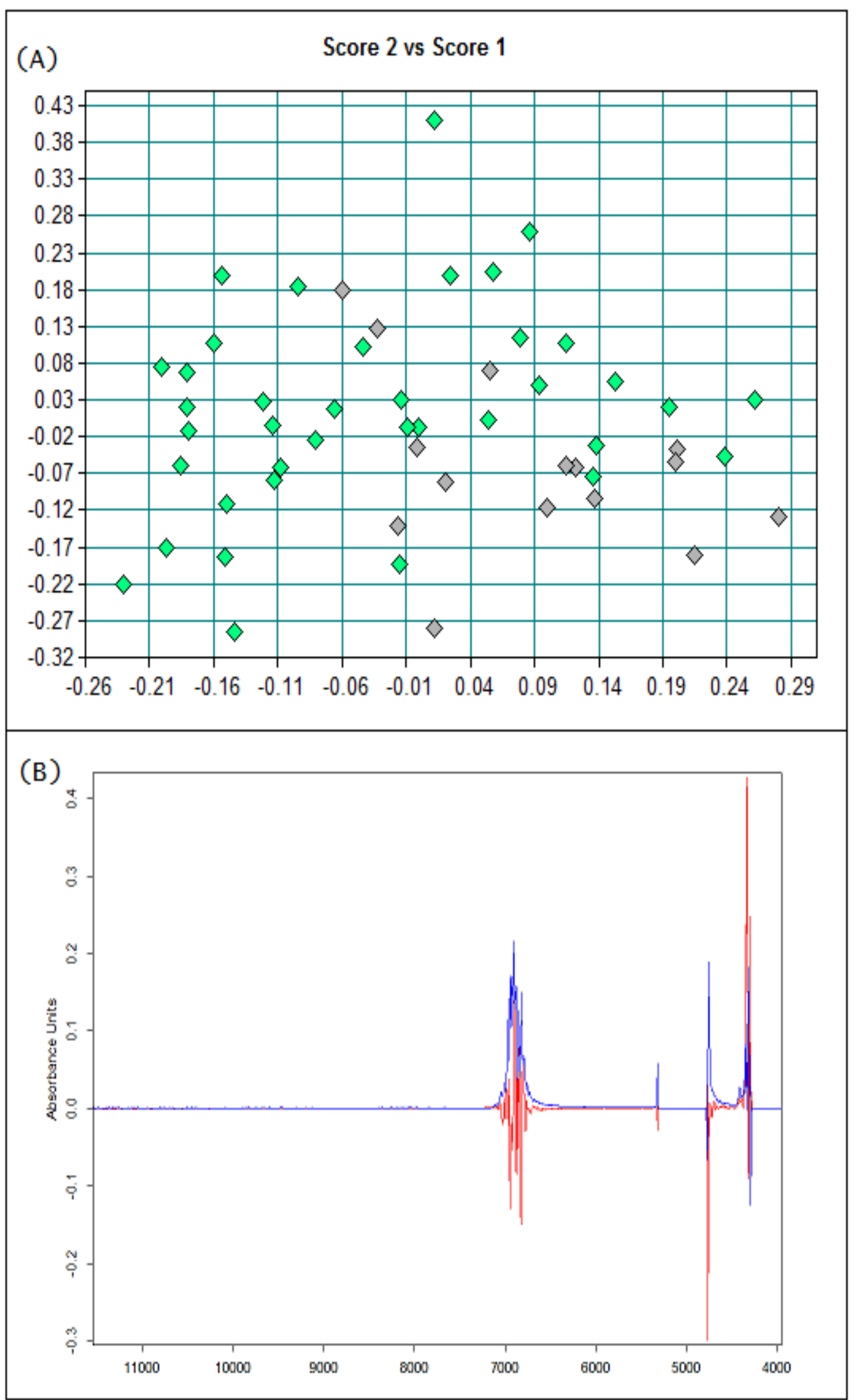

Figure 7. Mono varietal PCA plot of Primitivo samples from NIR data; (A) pure (in magenta) and mixed (in orange); (B) PCA loadings. 


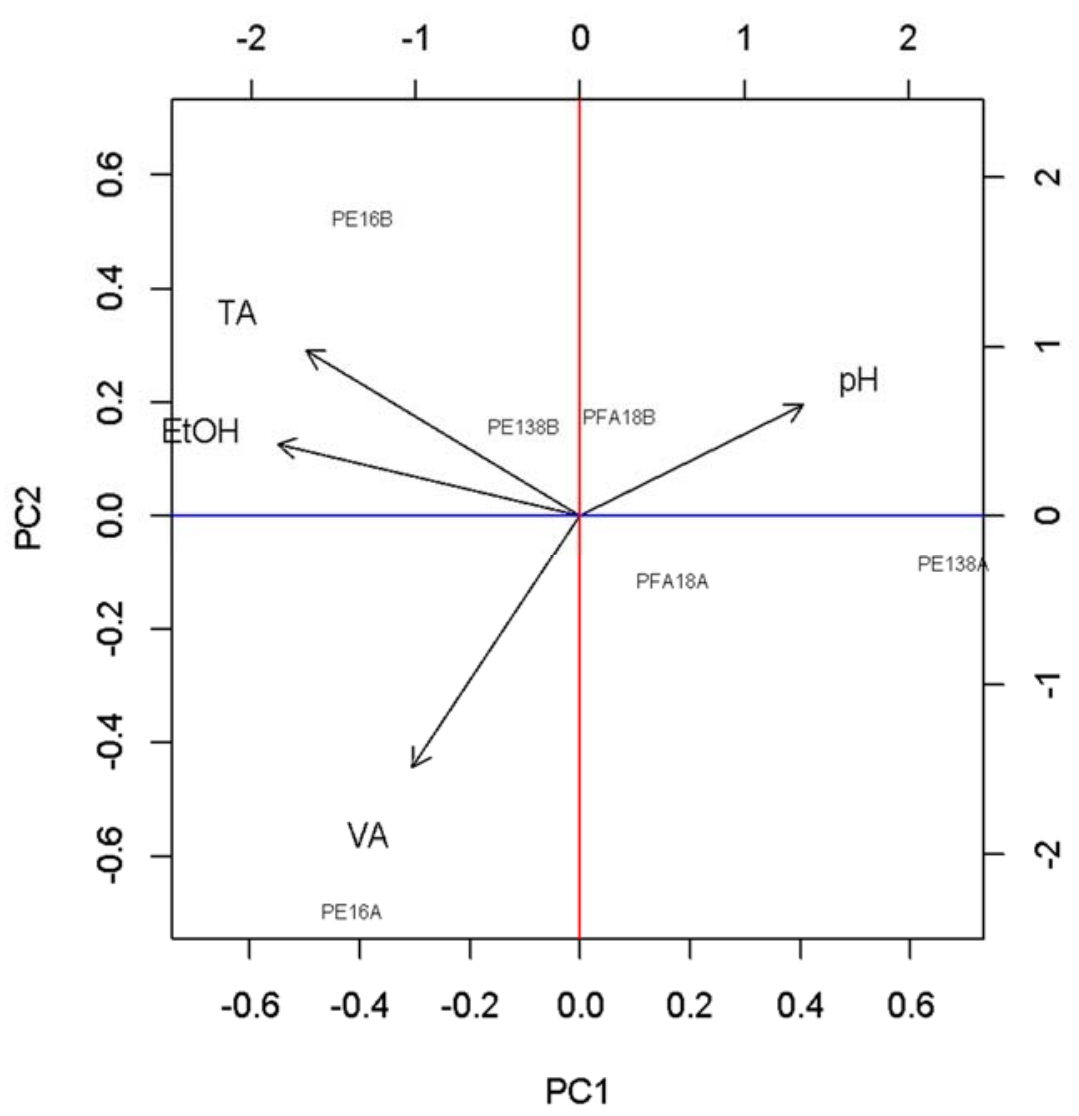

Figure 8. Mono varietal PCA plot of Primitivo (P) wines from chemical analyses.

\subsection{Sensory Analysis}

The sensory analysis performed (Table 2) showed significant differences only for fruity fresh, fruity mature, and appreciation descriptors. These results show how the sensory profiles of Aleatico nero wines obtained with pure or mixed fermentations do not differ substantially. A possible explanation for this lack of appreciable differences in the sensory profiles could be ascribed to the small or subtle influence of the $S$. bacillaris in the fermentation process, which was probably lead by the S. cerevisiae strains. Wines with different chemical compositions should differ significantly in terms of sensory attributes, therefore this lack of significant differences found by testers could explain the small or non-existent differentiation found with NIR analysis for those wines.

Table 2. Sensory analysis results (cv. Aleatico nero).

\begin{tabular}{cccccccc}
\hline Attributes & FA18A & E138B & E16B & E16A & E138A & FA18B & Significance \\
\hline Colour Intensity & 6 & 7 & 6 & 4 & 6 & 6 & n.s. \\
Fruity (Fresh) & $1 \mathrm{~b}$ & $4 \mathrm{ab}$ & $4 \mathrm{ab}$ & $6 \mathrm{ab}$ & $4 \mathrm{ab}$ & $0 \mathrm{~b}$ & $* *$ \\
Fruity (Mature) & $1 \mathrm{ab}$ & $3 \mathrm{ab}$ & $2 \mathrm{a}$ & $3 \mathrm{ab}$ & $3 \mathrm{a}$ & $0 \mathrm{~b}$ & $*$ \\
Floral & 1 & 2 & 2 & 2 & 1 & 0 & n.s. \\
Herbal & 1 & 2 & 1 & 2 & 1 & 0 & n.s. \\
Spicy & 1 & 4 & 3 & 2 & 2 & 0 & n.s. \\
Acidity & 7 & 7 & 7 & 6 & 6 & 7 & n.s. \\
Astringency & 7 & 5 & 4 & 4 & 5 & 3 & n.s. \\
Body & 4 & 4 & 4 & 5 & 5 & 2 & n.s. \\
Alcohol & 6 & 6 & 6 & 6 & 7 & 4 & n.s. \\
Persistency & 4 & 5 & 4 & 5 & 4 & 4 & n.s. \\
Appreciation & $2 \mathrm{bc}$ & $5 \mathrm{a}$ & $4 \mathrm{ab}$ & $6 \mathrm{a}$ & $4 \mathrm{a}$ & $0 \mathrm{c}$ & $* * *$ \\
\hline
\end{tabular}

n.s.: not significant; ${ }^{*} p \leq 0.05 ;{ }^{* *} p \leq 0.01 ; * * * \leq 0.001$. Values bearing the same letter in the same row are not significantly different from each other (Kruskal-Wallis test $p \leq 0.05$ and Dunn's post-hoc test). 


\section{Discussion}

The need to accurately predict the results of the fermentation process has led to the progressive abandonment of spontaneous fermentations in favor of the use of selected S. cervisiae strains. This choice has led to a flattening of the taste of wines over time. This problem can be overcome by using selected non-Saccharomyces yeast strains that are able to give particular aromas to the wine. Among non-Saccharomyces yeasts, the $S$. bacillaris, which has been isolated from grapes, winery environments, and during spontaneous fermentations in different countries in the world, looks like a very promising candidate. Indeed, it shows many positive characteristics, such as low ethanol and high glycerol production and fructophily (i.e., ability to utilize fructose preferentially when fructose and glucose are available in the environment). Therefore, its use together with selected $S$. cerevisiae in mixed culture fermentations has been proposed to increase wine characteristics appreciated by consumers [24]. In the present work twelve winemakings of well-known wine grape varieties, namely Negramaro, Primitivo, and Aleatico nero, have been performed with starter cultures of S. cerevisiae, both pure or in combination with $S$. bacillaris yeast. The wines produced have been analyzed for basic chemical parameters and by NIR spectroscopy. The comparison of these analyses showed how the difference among wines is strongly influenced by compounds with higher concentrations, such as ethanol. NIR spectroscopy was able to detect differences in the metabolomic profile of wines with similar ethanol content, which could be attributed to the different winemaking methods used (i.e., pure or mixed fermentation). A direct investigation of the NIR spectra regions responsible for the differentiation of wines, with close ethanol content, allowed for the attribution of the differentiation to compounds such as glycerol.

It was found that NIR analysis did not effectively discriminate between wines obtained with pure or mixed fermentations for all the varieties tested. In order to understand if this was due to a lack of discriminant capacity of the NIR analysis or to a lack of significant differences in wine composition, a sensory analysis was performed. Sensory analysis is commonly employed to check for differences in wine composition. With this type of analysis, it is possible to evaluate similarities and differences among wines which are actually linked to the actual chemical composition of each sample $[25,26]$. We found that when NIR technique was not able to discriminate among wines obtained with different fermentation procedures, the sensory analysis performed on the same set of wines confirmed that those samples were not perceived as different. The lack of significant differences in terms of compounds responsible for the perceived sensory attributes tested confirms the small differentiation found with NIR analysis for those wines. The alcoholic fermentation of grape musts is a rather complex process that involves the sequential development of microorganisms and their interaction. In the mixed fermentation winemaking, the fermentation process was probably led by S. cerevisiae, with small or no appreciable influence of the non-Saccharomyces yeast.

Instead of performing a quantitative analysis, which requires a large number of samples to be analyzed by primary techniques and several steps of optimization which are necessary to build a calibration curve, in this work we tested the ability of NIR technique to discriminate among samples without any a priori knowledge of their composition. Samples which did not differ by NIR analysis also did not show differences by sensory analysis. The preliminary results obtained from this study show how it is possible, with a fast and economic procedure, to check if there are differences in the final products obtained with different winemaking procedures. If the results of a preliminary NIR analysis step are not positive, the efforts (and costs) related to further analyses can be saved. This is especially useful when dealing with a large number of samples since it avoids the need for more detailed analysis, saving both money and time.

Author Contributions: Conceptualization, M.F.C. and T.B.; data curation, V.A.; formal analysis, M.V. and T.B.; statistical analysis, V.A.; funding acquisition, D.A.; supervision, R.P. and D.A.; writing—original draft, T.B.; writing-review and editing, A.D.M.

Funding: This research was funded by the International Organisation of Vine and Wine, OIV 2017 research grant. 
Acknowledgments: This work was supported by OIV 2017 research grant. The yeasts have been kindly provided by Professor Hervé, Institut Universitaire de la Vigne et du Vin, University of Burgundy, Dijon, France.

Conflicts of Interest: The authors declare no conflict of interest. The founding sponsors had no role in the design of the study; in the collection, analyses, or interpretation of data; in the writing of the manuscript, and in the decision to publish the results.

\section{References}

1. World Vitiviniculture Situation. OIV Statistical Report on World Vitiviniculture. 2017. Available online: http:/ / www.oiv.int/public/medias/5479/oiv-en-bilan-2017.pdf (accessed on 3 September 2018).

2. Fleet, G.H. Yeast interactions and wine flavour. Int. J. Food Microb. 2003, 86, 11-22. [CrossRef]

3. Ciani, M.; Capece, A.; Comitini, F.; Canonico, L.; Siesto, G.; Romano, P. Yeast interactions in inoculated wine fermentation. Front. Microbiol. 2016, 7, 555. [CrossRef] [PubMed]

4. Comitini, F.; Gobbi, M.; Domizio, P.; Romani, C.; Lencioni, L.; Mannazzu, I.; Ciani, M. Selected non-Saccharomyces wine yeasts in controlled multistarter fermentations with Saccharomyces cerevisiae. Food Microbiol. 2011, 28, 873-882. [CrossRef] [PubMed]

5. Tofalo, R.; Schirone, M.; Torriani, S.; Rantsiou, K.; Cocolin, L.; Perpetuini, G.; Suzzi, G. Diversity of Candida zemplinina strains from grapes and Italian wines. Food Microbiol. 2012, 29, 18-26. [CrossRef] [PubMed]

6. Magyar, I.; Tóth, T. Comparative evaluation of some oenological properties in wine strains of Candida stellata, Candida zemplinina, Saccharomyces uvarum and Saccharomyces cerevisiae. Food Microbiol. 2011, 28, 94-100. [CrossRef] [PubMed]

7. Englezos, V.; Rantsiou, K.; Cravero, F.; Torchio, F.; Ortiz-Julien, A.; Gerbi, V.; Rolle, L.; Cocolin, L. Starmerella bacillaris and Saccharomyces cerevisiae mixed fermentations to reduce ethanol content in wine. Appl. Microbiol. Biotechnol. 2016, 100, 5515-5526. [CrossRef] [PubMed]

8. Englezos, V.; Torchio, F.; Cravero, F.; Marengo, F.; Giacosa, S.; Gerrbi, V.; Rantsiou, K.; Rolle, L.; Cocolin, L. Aroma profile and composition of Barbera wines obtained by mixed fermentations of Starmerella bacillaris (synonym Candida zemplinina) and Saccharomyces cervisiae. LWT Food Sci. Technol. 2016, 73, 567-575. [CrossRef]

9. Martelo-Vidal, M.J.; Vázquez, M. Evaluation of Ultraviolet, Visible, and Near Infrared Spectroscopy for the Analysis of Wine Compounds. Czech J. Food Sci. 2014, 32, 37-47. [CrossRef]

10. Gishen, M.; Dambergs, R.G.; Cozzolino, D. Grape and wine analysis-Enhancing the power of spectroscopy with chemometrics. A review of some applications in the Australian wine industry. Aust. J. Grape Wine Res. 2005, 11, 296-305. [CrossRef]

11. Cozzolino, D.; Dambergs, R.G.; Janik, L.; Cynkar, W.U.; Gishen, M. Analysis of grapes and wine by near infrared spectroscopy. J. Near Infrared Spectrosc. 2006, 14, 279-289. [CrossRef]

12. López, M.G.; Sarahí García-González, A.; Franco-Robles, E. Carbohydrate Analysis by NIRS-Chemometrics. In Developments in Near-Infrared Spectroscopy; Kyprianidis, K., Ed.; IntechOpen: London, UK, 2017; pp. 81-95. Available online: https://www.intechopen.com/books/developments-in-near-infrared-spectroscopy/ carbohydrate-analysis-by-nirs-chemometrics (accessed on 3 September 2018).

13. Garcia-Jares, C.M.; Médina, B. Application of multivariate calibration to the simultaneous routine determination of ethanol, glycerol, fructose, glucose and total residual sugars in botrytized-grape sweet wines by means of near-infrared reflectance spectroscopy. Fresenius J. Anal. Chem. 1997, 357, 86-91. [CrossRef]

14. Di Egidio, V.; Sinelli, N.; Giovanelli, G.; Moles, A.; Casiraghi, E. NIR and MIR spectroscopy as rapid methods to monitor red wine fermentation. Eur. Food Res. Technol. 2010, 230, 947-955. [CrossRef]

15. ISO 3591. Sensory Analysis_-Apparatus_Wine-Tasting Glass; International Organization for Standardization: Geneva, Switzerland, 1977.

16. ISO 6658. Sensory Analysis—Methodology_General Guidance; International Organization for Standardization: Geneva, Switzerland, 2005.

17. ISO 8598. Sensory Analysis—General Guidance for the Design of Test Rooms; International Organization for Standardization: Geneva, Switzerland, 2007.

18. ISO 4121. Sensory Analysis-Guidelines for the Use of Quantitative Response Scales; International Organization for Standardization: Geneva, Switzerland, 2003. 
19. Meilgaard, M.; Civille, G.V.; Carr, B.T. Sensory Evaluation Techniques, 3rd ed.; CRC Press: Boca Raton, FL, USA, 1999; ISBN 0849302765.

20. Conzen, J.P. Multivariate Calibration. A Practical Guide for Developing Methods in the Quantitative Analytical Chemistry, 3rd ed.; Bruker Optik GmbH: Ettlingen, Germany, 2014; ISBN 978-3-929431-13-1.

21. Workman, J., Jr.; Weyer, L. Chapter 6 Water. In Practical Guide and Spectral Atlas for Interpretive Near-Infrared Spectroscopy, 2nd ed.; CRC Press Taylor and Francis Group: Boca Raton, FL, USA, 2012; pp. 55-63, ISBN 9781439875254.

22. Queji, M.D.; Wosiacki, G.; Cordeiro, G.A.; Peralta-Zamora, P.G.; Nagata, N. Determination of simple sugars, malic acid and total phenolic compounds in apple pomace by infrared spectroscopy and PLSR. Int. J. Food Sci. Technol. 2010, 45, 602-609. [CrossRef]

23. Masneuf-Pomarede, I.; Juquin, E.; Miot-Sertier, C.; Renault, P.; Laizet, Y.; Salin, F.; Alexandre, H.; Capozzi, V.; Cocolin, L.; Colonna-Ceccaldi, B.; et al. The yeast Starmerella bacillaris (synonym Candida zemplinina) shows high genetic diversity in winemaking environments. FEMS Yeast Res. 2015, 15, fov045. [CrossRef] [PubMed]

24. Englezos, V.; Giacosa, S.; Rantsiou, K.; Rolle, L.; Cocolin, L. Starmerella bacillaris in winemaking: Opportunities and risks. Curr. Opin. Food Sci. 2017, 17, 30-35. [CrossRef]

25. Lawless, H.T.; Heymann, H. Sensory Evaluation of Food: Principles and Practices, 2nd ed.; Springer: New York, NY, USA, 2010.

26. Varela, P.; Ares, G. Sensory profiling, the blurred line between sensory and consumer science. A review of novel methods for product characterization. Food Res. Int. 2012, 48, 893-908. [CrossRef]

(C) 2018 by the authors. Licensee MDPI, Basel, Switzerland. This article is an open access article distributed under the terms and conditions of the Creative Commons Attribution (CC BY) license (http:/ / creativecommons.org/licenses/by/4.0/). 